\title{
Exchange energy density in density-functional theory via the Dirac density matrix for a nonrelativistic 10-electron atomic ion compared with Becke's proposal for a gradient-corrected local-density-approximation result
}

\author{
M. L. Glasser* \\ Department of Physics, Clarkson University, Potsdam, New York 13699-5820, USA \\ N. H. March \\ Department of Physics, University of Antwerp, Groenenborgerlaan 171, 2020 Antwerp, Belgium; \\ University of Oxford, Oxford, England, United Kingdom and International Centre for Theoretical Physics, Strada Costiera, 11, \\ Miramare, Trieste, Italy \\ L. M. Nieto \\ Departamento de Física Teórica, Atómica y Óptica, Universidad de Valladolid, 47071 Valladolid, Spain
}

(Received 11 December 2008; published 13 April 2009)

\begin{abstract}
Especially in atomic systems, it is now well established that exchange energy generally dominates correlation effects. Therefore we focus here on the exchange energy density $\varepsilon_{x}(r)$ as given in terms of the idempotent Dirac density matrix. This is then brought into contact with the one-parameter form of Becke's functional, which corrects the local-density-approximation form $-c_{x}[n(r)]^{4 / 3}$ with $n(r)$ as the ground-state electron density, $c_{x}=(3 / 4) e^{2}(3 / \pi)^{1 / 3}$, by terms involving the dimensionless gradient ratio $|\nabla n(r)| / n^{4 / 3}(r)$. A particular nonrelativistic model of the 10-electron Ne-like atomic ions, with large atomic number $Z$, is then compared to Becke's approximation to $\varepsilon_{x}(r)$.
\end{abstract}

DOI: $10.1103 /$ PhysRevA.79.042506

PACS number(s): 31.15.E-, 03.75.Ss, 05.30.Fk, 73.21.La

\section{BACKGROUND}

It is now well established for neutral atoms that the exchange energy generally dominates, numerically, the correlation contribution [1]. Therefore there has been lately a resurgence of interest in the exchange energy $E_{x}$ and the corresponding exchange potential $V_{x}(\boldsymbol{r})$ in density-functional theory (DFT).

A notable contribution in the above context is that of Della Sala and Görling [2], referred to here as DG who, on the basis of the somewhat drastic assumption that the Hartree-Fock determinant equaled its Kohn-Sham counterpart, derived an integral equation for the exchange potential $V_{x}(\boldsymbol{r})$. Howard and March [3] subsequently gave a formally exact generalization of the DG result, but it involved a function they denoted by $P(\boldsymbol{r})$, for which only the sum rule

$$
\int P(\boldsymbol{r}) d \boldsymbol{r}=0
$$

is known.

\section{EXCHANGE ENERGY IN TERMS OF THE IDEMPOTENT DIRAC DENSITY MATRIX}

In terms of the Slater-Kohn-Sham (SKS) [4,5] orbitals $\psi_{i}(\boldsymbol{r})$ of DFT, one defines the ground-state Dirac density matrix $\gamma\left(\boldsymbol{r}, \boldsymbol{r}^{\prime}\right)$ as [6]

\footnotetext{
*laryg@ clarkson.edu

†luismi@metodos.fam.cie.uva.es
}

$$
\gamma\left(\boldsymbol{r}, \boldsymbol{r}^{\prime}\right)=\sum_{\mathrm{occ}} \psi_{i}^{*}(\boldsymbol{r}) \psi_{i}\left(\boldsymbol{r}^{\prime}\right),
$$

which is clearly related to the (formally exact) ground-state electron density $n(\boldsymbol{r})$ by

$$
n(\boldsymbol{r})=\left.\gamma\left(\boldsymbol{r}, \boldsymbol{r}^{\prime}\right)\right|_{\boldsymbol{r}=\boldsymbol{r}^{\prime}} .
$$

Then, as was first written by Dirac [6], the total exchange energy, $E_{x}$ say, is given by

$$
E_{x}=-\frac{e^{2}}{4} \int \frac{\left[\gamma\left(\boldsymbol{r}, \boldsymbol{r}^{\prime}\right)\right]^{2}}{\left|\boldsymbol{r}-\boldsymbol{r}^{\prime}\right|} d \boldsymbol{r} d \boldsymbol{r}^{\prime} .
$$

Below, we shall focus most attention on the exchange energy density, denoted by $\varepsilon_{x}(\boldsymbol{r})$. Although its definition is not unique, all choices must clearly satisfy the relation

$$
E_{x}=\int \varepsilon_{x}(\boldsymbol{r}) d \boldsymbol{r} .
$$

The most natural choice for exchange energy density is then, from Eqs. (4) and (5), given by

$$
\varepsilon_{x}(\boldsymbol{r})=-\left(e^{2 / 4}\right) \int \frac{\left|\gamma\left(\boldsymbol{r}, \boldsymbol{r}^{\prime}\right)\right|^{2}}{\left|\boldsymbol{r}-\boldsymbol{r}^{\prime}\right|} d \boldsymbol{r}^{\prime},
$$

which Slater [4] adopted (also see Kleinman [7]). We will mainly use this in the present paper; however, in Appendix A an alternative form is given which can be shown to be a functional of the electron density $n(r)$.

Let us consider now a 10-electron "atomic ion" model in the nonrelativistic limit of large nuclear charge. The exchange energy density $\varepsilon_{x}(\boldsymbol{r})$ in exact analytical form for an atomic model studied by Howard et al. [8] will be exten- 


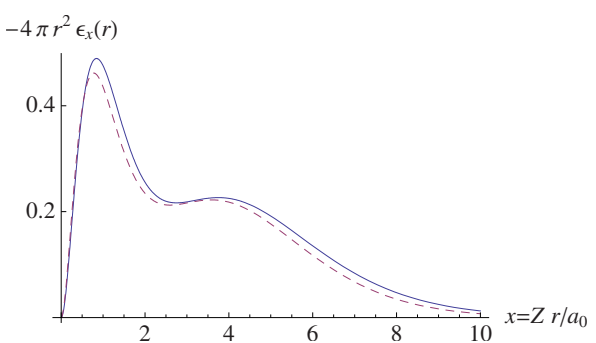

FIG. 1. (Color online) Comparison of $-4 \pi r^{2} \varepsilon_{x}(r)$ (in units of $e^{2} Z^{4} / a_{0}^{4}$ ) for Howard et al. (2.2) of [8] (solid curve) and LDA (dashed curve) calculated from Eq. (7). Horizontal axis: $x=Z r / a_{0}$.

sively developed below. This describes a 10-electron Ne-like nonrelativistic atomic ion in the limit of large atomic number $Z$. Here the wave functions are hydrogenic, and the Dirac density matrix entering Eq. (6) for the exchange energy density $\varepsilon_{x}(\boldsymbol{r})$ is given analytically in the paper of March and Santamaria [9]. First we used the exact (hydrogenic) groundstate density $n(\boldsymbol{r})$ as given in [8] to plot $4 \pi r^{2} \varepsilon_{x}(\boldsymbol{r})$ versus $r$ in Fig. 1, for the atomic number $Z=92$. Howard et al. [8] used the analytical density matrix $\gamma\left(\boldsymbol{r}, \boldsymbol{r}^{\prime}\right)$ of [9], which allowed $\varepsilon_{x}(\boldsymbol{r})$ to be obtained in exact analytical form for this model when inserted in Eq. (5). The asymptotic form at large $r$ given by one of us [10], namely, $\varepsilon_{x}(r)$ which tends to $-\left(e^{2} / 2 r\right) n(r)$, is also plotted for comparison in Fig. 2.

\section{COMPARISON OF THE SLATER-DIRAC FORMULA (6) WITH A BECKE-TYPE GRADIENT- CORRECTED LOCAL-DENSITY-APPROXIMATION RESULT}

Following the early proposal of Herman et al. [11] (see also [12]), Becke [13] proposed to correct Dirac's result [6]

$$
\varepsilon_{x}^{\mathrm{LDA}}(\boldsymbol{r})=-c_{x}\{n(\boldsymbol{r})\}^{4 / 3}, \quad c_{x}=\frac{3}{4} e^{2}(3 / \pi)^{1 / 3}
$$

with a "partial sum" to infinite order of gradient terms of the form $|\nabla n(\boldsymbol{r})| / n(\boldsymbol{r})^{4 / 3}$. Rather than focusing on the details of Becke's one-parameter expression [13], we shall utilize below the exact analytical form of $\varepsilon_{x}(r)$ at large $Z$, which we take throughout in all numerical examples to be 92 , to test the Becke-type gradient expansion. We embody Becke's ansatz in the proposal that for this particular 10-electron model

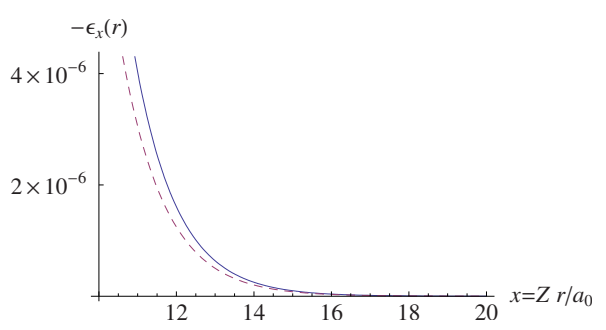

FIG. 2. (Color online) Comparison of $-\varepsilon_{x}(r)$ (in units of $\left.e^{2} Z^{4} / a_{0}^{4}\right)$ taken from Eq. (3) of [8] (solid curve) and $\left(e^{2} / 2 r\right) n(r)$ (dashed curve) for large $r$. Horizontal axis: $x=Z r / a_{0}$.

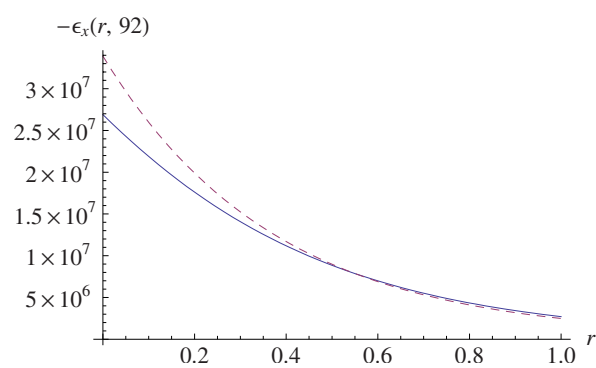

FIG. 3. (Color online) $\varepsilon_{x}(r, 92)$ (dashed curve) and $\varepsilon_{x}^{\mathrm{LDA}}(r, 92)$ (solid curve) versus $r$ (in a.u.).

$$
\varepsilon_{x}(r, Z)-\varepsilon_{x}^{\mathrm{LDA}}(r, Z)=\Delta_{x}\left(|\nabla n| / n^{4 / 3}\right)
$$

and also define for use below

$$
y(r, Z)=[n(r)]^{4 / 3} /|\nabla n| .
$$

Both $\varepsilon_{x}(r, 92)$ and $\varepsilon_{x}^{\mathrm{LDA}}(r, 92)$ have been plotted in Fig. 3 .

In Fig. 4, motivated by Becke's assumption set out above, we plot $y(r, Z)$ defined in Eq. (9) versus $r$ for $Z=92$, by inserting the March-Santamaria [9] density $n(r)$. We note first that, for $y>y_{c}=0.23, r$ is a multiple-valued function of $y$. Thus, as demonstrated explicitly in Fig. 5, for $r>r_{c}$, it is possible to extract $\varepsilon_{x}(y)$ uniquely; the multivalued nature of $r$ for $y>y_{c}$ makes it quite clear that the Howard et al. form [8] of $\varepsilon_{x}-\varepsilon_{x}^{\mathrm{LDA}}$ is a nonlocal functional of $y$ in Eq. (9) (see also [12]), even if higher derivatives of the density are ignored. We turn next from the exchange energy density to the much more difficult problem of the exchange potential $V_{x}(r)$ in the 10 -electron atomic ion model.

\section{EXCHANGE POTENTIAL FOR A NONRELATIVISTIC 10-ELECTRON ATOMIC ION IN THE LIMIT OF LARGE ATOMIC NUMBER $Z$}

To date, the functional derivative of $\varepsilon_{x}$ with respect to density, i.e., the exchange potential $V_{x}(r)$, is not known analytically for the 10-electron model for large $Z$. However, it is available numerically from the work of Howard et al. [14] (see Fig. 1 labeled: Coulomb: first iteration) who used the optimized exchange potential (OEP) method.

Here, therefore, we shall bring this precise numerical result for $V_{x}(r)$ for this model into contact with the parts of $V_{x}(r)$ already known which we summarize below. Dividing

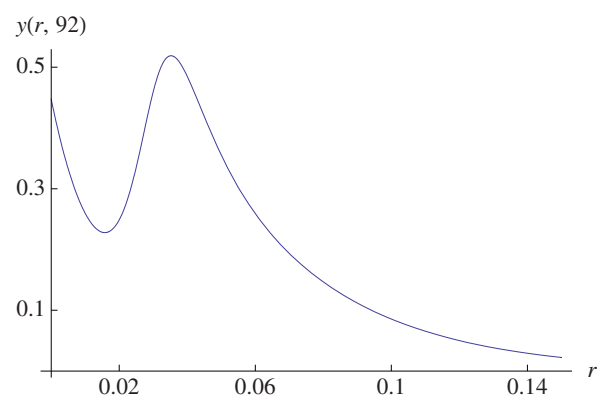

FIG. 4. (Color online) Dimensionless gradient ratio $y(r, 92)$ defined in Eq. (9) versus $r$ in atomic units. The quantity $y$ is monotonic for $r>r_{c}=0.64$. 


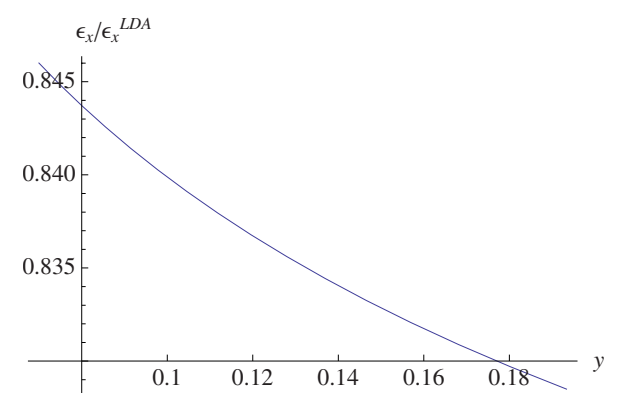

FIG. 5. (Color online) Dimensionless ratio $\varepsilon_{x} / \varepsilon_{x}^{\mathrm{LDA}}$ versus $y$ for $y$ in the monotonic region in Fig. 4 .

$E_{x}$ into the sum of three pieces, we have [12]

$$
\begin{gathered}
V_{x}^{(1)}(r)=\frac{\delta E_{x}^{(1)}}{\delta n(r)}=-\frac{10 \pi e^{2}}{3} r^{2} n(r), \\
V_{x}^{(2)}(r)=\frac{\delta E_{x}^{(2)}}{\delta n(r)}=\frac{70 \pi e^{2}}{9} r^{2} n(r)+\frac{28 \pi e^{2}}{9} r^{3} n^{\prime}(r) \\
+\frac{7 \pi e^{2}}{27} r^{4} n^{\prime \prime}(r)+\frac{56 \pi e^{2}}{9} r^{4} F(r)-I^{(2)}(r),
\end{gathered}
$$

where all but the last term in (11) can be evaluated explicitly, since $n(r)$ and $F(r)$ are known functions of $Z$ and $r$. However, the last term is given by

$$
I^{(2)}(r)=\frac{224 \pi^{2} e^{2} m}{27 \hbar^{2}} \int_{0}^{\infty} d s n(s) s^{6} \frac{\delta t(s)}{\delta n(r)}
$$

and, unfortunately, the functional derivative in (12) is not yet known completely for this model. (Only the contributions to it from the $1 s$ and $2 s$ electrons have been estimated [8].)

The third, and final, contribution $V_{x}^{(3)}(r)$ to the desired exchange potential $V_{x}(r)$ involves the same functional derivative of $t(s)$ and is given by

$$
\begin{aligned}
V_{x}^{(3)}(r)= & -\frac{112 \pi e^{2}}{3} r^{4} F(r)-\frac{32 \pi e^{2}}{3} r^{5} F^{\prime}(r)-\frac{2 \pi e^{2}}{3} r^{6} F^{\prime \prime}(r) \\
& +J^{(3)}(r),
\end{aligned}
$$

where

$$
J^{(3)}(r)=\frac{64 \pi^{2} e^{2} m}{3 \hbar^{2}} \int_{0}^{\infty} d s s^{2} F(s) \frac{\delta t(s)}{\delta n(r)} .
$$

The quantities $I^{(2)}(r)$ and $J^{(3)}(r)$ are discussed further below.

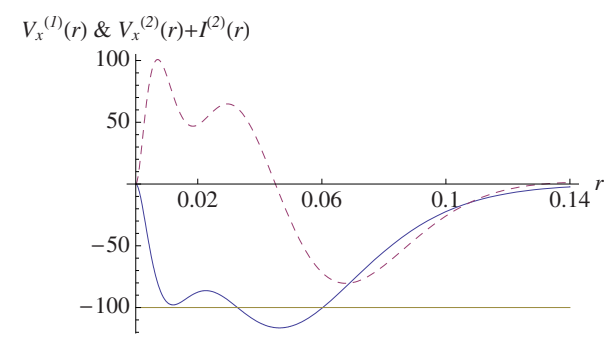

FIG. 6. (Color online) $V_{x}^{(1)}(r)$ (solid curve) and $V_{x}^{(2)}(r)+I^{(2)}(r)$ (dashed curve) versus $r$ (a.u.).

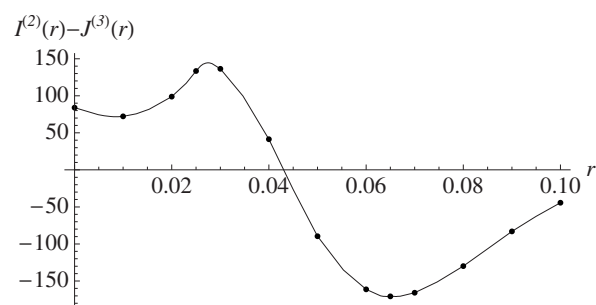

FIG. 7. The functional integral $I^{(2)}(r)-J^{(3)}(r)$ versus $r$ (a.u.).

Figure 6 shows the exchange contribution $V_{x}^{(1)}(r)$ versus $r$ for the 10 -electron atomic ion with $Z=92$. It is, in essence, the negative of the March-Santamaria radial ground-state density $4 \pi r^{2} n(r)$. Although it falls off at large $r$ much more rapidly than the OEP $V_{x}(r)$, it is encouraging that it shows "structure" in the same range of $r$ as for the OEP result. However, it also heralds the fact that the OEP $V_{x}(r)$ reflects a rather sensitive interplay between the known $V_{x}^{(1)}(r)$ and the incompletely known $V_{x}^{(2)}$ and $V_{x}^{(3)}$ and therefore it seemed to be of interest to plot from Eq. (11) the quantity $V_{x}^{(2)}+I^{(2)}$ in Fig. 6. By using the OEP $V_{x}(r)$, Fig. 7 shows a rough plot of $I^{(2)}-J^{(3)}(r)$ since $V_{x}(r)$ is only available at present to graphical accuracy.

\section{Exchange force $-\partial V_{x} / \partial r$}

As discussed in more detail in Sec. V, the total exchange energy $E_{x}$ is related to the exchange force $-\partial V_{x} / \partial r$ by a virial-like relation. Furthermore, Howard et al. [14] also plot $-\partial V_{x} / \partial r$ versus $r$ for $Z=92$ and this graph shows a number of distinctive features. In Fig. 8 we compare $-\partial V_{x}^{(1)}(r) / \partial r$ with points from the Coulomb curve in Fig. 3 of [14]. Again, as with the exchange energy density, the correct $V_{x}(r)$ is the result of a precise balance among the components $V_{x}^{(i)}(r)$, where $i=1,2,3$.

\section{USE OF A LEVY-PERDEW VIRIAL-LIKE RELATION BETWEEN $E_{x}$ AND THE EXCHANGE POTENTIAL $V_{x}(r)$}

Levy and Perdew [15] derived the important virial-like relation between the total exchange energy $E_{x}$ and the exchange potential $V_{x}(r)$,

$$
E_{x}=-\int n(\boldsymbol{r}) \boldsymbol{r} \cdot \nabla V_{x}(\boldsymbol{r}) d \boldsymbol{r} .
$$

Later, Nagy and March [16] performed an integration by parts on (15), setting the resulting contribution from the lim-

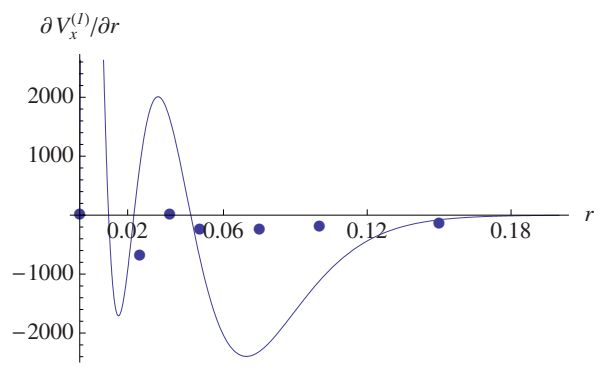

FIG. 8. (Color online) $-\partial V_{x}^{(1)}(r) / \partial r$ versus $r$ (curve). The dots are results from [14] Fig. 2 ( $Z=92)$. 
its to zero on physical grounds and obtained the result

$$
E_{x}=\int n(\boldsymbol{r}) V_{x}(\boldsymbol{r})\left[3+\boldsymbol{r} \cdot \frac{\nabla n(\boldsymbol{r})}{n(\boldsymbol{r})}\right] d \boldsymbol{r} .
$$

Let us consider now the application to 10-electron atomic ion model, where spherical symmetry reduces Eq. (15) to the form

$$
E_{x}=-\int n(r) r \frac{\partial V_{x}}{\partial r} d \boldsymbol{r} .
$$

In Appendix $\mathrm{A}$, the exchange energy is expressed as $E_{x}$ $=\Sigma_{i=1}^{3} E_{x}^{(i)}$ and hence, since $V_{x}=\delta E_{x} / \delta n(r)$, we find

$$
E_{x}=\sum_{i=1}^{3} E_{x}^{(i)}=-\sum_{i=1}^{3} \int n(r) r \frac{\partial V_{x}^{(i)}(r)}{\partial r} d \boldsymbol{r} .
$$

Since $E_{x}^{(1)}$ and $V_{x}^{(1)}(r)$ are known explicitly from Appendix A, Eq. (A6) and Sec. IV, Eq. (10), respectively, one has

$$
E_{x}^{(1)}=\int \varepsilon_{x}^{(1) A}(r) d \boldsymbol{r}=-\frac{5 \pi}{3} e^{2} \int r^{2} n(r) d \boldsymbol{r}
$$

and

$$
V_{x}^{(1)}=-\frac{10 \pi}{3} e^{2} r^{2} n(r)
$$

and we can use the explicit form of $n(r)$ to test whether Eq. (15) relates $E_{x}^{(1)}$ and $\partial V_{x}^{(1)} / \partial r$ or whether Eq. (15) only holds between $\sum_{i=1}^{3} E_{x}^{(i)}$ and $\sum_{i=1}^{3} V_{x}^{(i)}(r)$.

Since $\partial V_{x}^{(1)} / \partial r$ from Eq. (10) is given by

$$
\frac{\partial V_{x}^{(1)}}{\partial r}=-\frac{20 \pi e^{2}}{3} r n(r)-\frac{10 \pi e^{2}}{3} r^{2} n^{\prime}(r),
$$

we find

$$
-\int n(r) r \frac{\partial V_{x}^{(1)}}{\partial r} d \boldsymbol{r}=-\frac{422165}{972}=-434.326,
$$

which is precisely, for $Z=92$, in agreement with $E_{x}^{(1)}=$ $-(18355 / 3888) Z e^{2} / a_{0}$ as reported in [8].

\section{SUMMARY AND FUTURE DIRECTIONS}

An exact analytical form for the exchange energy density $\varepsilon_{x}(r)$ defined in Eq. (6) was derived in [3] for nonrelativistic

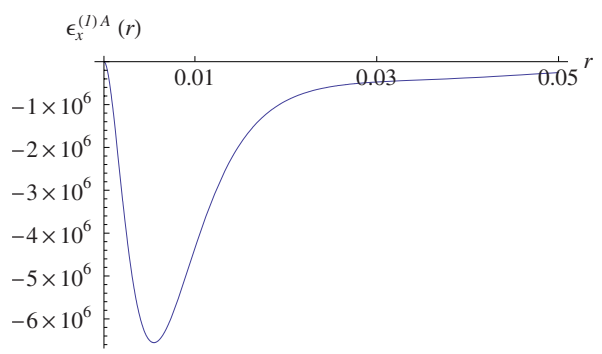

FIG. 9. (Color online) $\varepsilon_{x}^{(1) A}(r)$ versus $r$ (a.u.) in Eq. (A6) for $Z=92$.

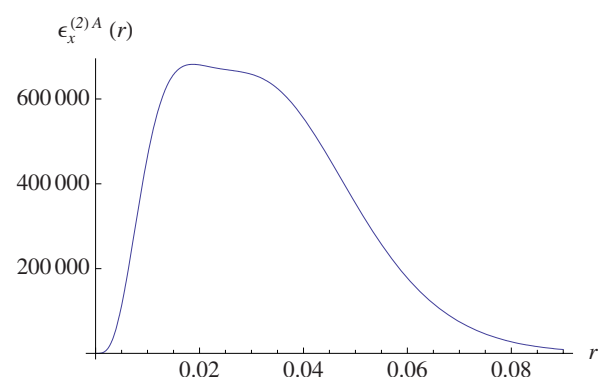

FIG. 10. (Color online) $\varepsilon_{x}^{(2) A}(r)$ versus $r$ (a.u) in Eq. (A7) for $Z=92$.

Ne-like atomic ions in the limit of large nuclear charge $\mathrm{Ze}$. Here we have attempted to gain insight into the densityfunctional form $\varepsilon_{x}[n]$ in two ways. The first approach was prompted by Fig. 1, which compares $4 \pi r^{2} \varepsilon_{x}(r)$ with the local-density-approximation (LDA) form proportional to the $4 / 3$ power of the ground-state electron density $n(r)$. Becke [13] proposed correcting the ratio $\varepsilon_{x}(r) / \varepsilon_{x}^{\mathrm{LDA}}(r)$ by regarding it as a function of the dimensionless gradient ratio $y^{-1}$ as defined in Eq. (9). To examine this we have plotted $y(r, Z)$ for the 10-electron atomic ion having $Z=92$ in Fig. 4 and it is clear that below the region of $y=y_{c}$, where there is multivalued behavior, $y$ decreases monotonically with increasing $r$, and in this range a Becke-type assumption can be made formally exact. However, the existence of the multivalued region shows that, in the 10-electron ion case, either one must take $\varepsilon_{x}(r) / \varepsilon_{x}^{\mathrm{LDA}}(r)$ to be a nonlocal functional of the Becke gradient ratio (see also [12]) or include higher derivatives of $n(r)$ than simply $\nabla n(r)$.

This then prompted the fuller investigation of the alternative form of exchange energy density $\varepsilon_{x}^{A}(r)=\varepsilon_{x}^{(1) A}+\varepsilon_{x}^{(2) A}$ $+\varepsilon_{x}^{(3) A}$, which is set out in some details in Appendix $\mathrm{A}$ and illustrated in Figs. 9-12, having the merit that, in a formally exact fashion, $\varepsilon_{x}^{A}[n]$ exists [3]. But, the kinetic-energy density $t(r)$ is involved through the function $F(r)$ given in Eq. (A8). This suggested examining the difference $\varepsilon_{x}(r)-\varepsilon_{x}^{A}(r)$ vs $r$ shown in Fig. 13. Unfortunately, we are unable at present to express this difference as a function of $n(r)$, although Fig. 14 indicates some definite shape similarities with the Laplacian of the density $\nabla^{2} n(r)$ which occurs as the difference between two common choices of kinetic-energy density.

We have next focused on the exchange potential $V_{x}(r)$ for the 10-electron ions. In Sec. IV this is expressed as the sum of three parts, i.e., $V_{x}(r)=\sum_{i=1}^{3} V_{x}^{(i)}(r)$. The first of these, in Eq.

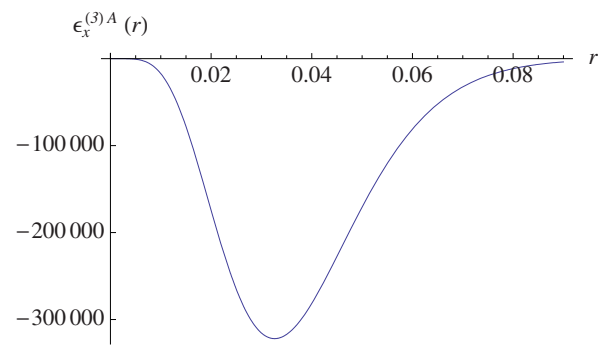

FIG. 11. (Color online) $\varepsilon_{x}^{(3) A}(r)$ versus $r$ (a.u.) in Eq. (A12) for $Z=92$. 


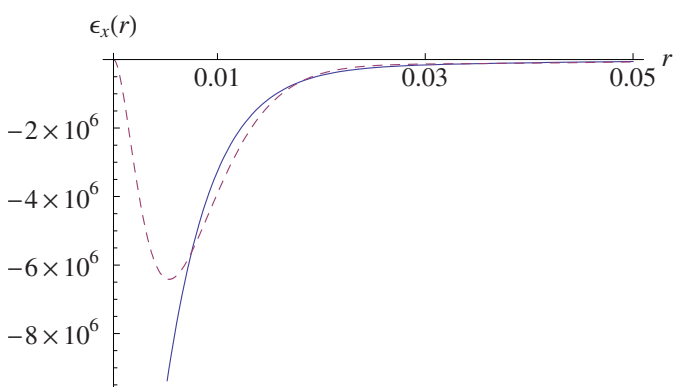

FIG. 12. (Color online) Comparison of $\varepsilon_{x}^{A}(r)$ (dashed curve) with Eq. (2.2) of [8] for $Z=92$.

(10), is essentially, in appropriate units, the radial density $4 \pi r^{2} n(r)$. Unfortunately $V_{x}^{(2)}(r)$ in Eq. (11) and $V_{x}^{(3)}(r)$ in Eq. (13) involve the kinetic-energy functional through $I^{(2)}(r)$ in Eq. (12) and $J^{(3)}(r)$ in Eq. (14). The difference $I^{(2)}(r)$ $-J^{(3)}(r)$ entering the total exchange potential $V_{x}(r)$ can fortunately be extracted from the OEP calculation made earlier by Howard et al. [14]; the result is displayed in Fig. 7. What all this shows is that the OEP result for $V_{x}(r)$ comes from a very delicate interplay among the three terms $V_{x}^{(i)}$, where $i$ $=1,2,3$ (see also Fig. 8). The first term, $V_{x}^{(1)}(r)$ has the nice property that, when related to the corresponding exchange energy contribution $E_{x}^{(1)}$, given just below Eq. (22), $E_{x}^{(1)}$ and $V_{x}^{(1)}(r)$ are precisely connected via the Levy-Perdew viriallike result (15).

Turning toward future work, it is clearly important to gain further insight into the kinetic-energy density $t$ in order to attack the functional derivative $\delta t(s) / \delta n(r)$ entering the exchange potential through Eqs. (11)-(14). Further consideration should naturally, in the future, be given to nonlocal extensions of Becke's result for the ratio $\varepsilon_{x} / \varepsilon_{x}^{\text {LDA }}$. The functional derivative, of course, of any approximate exchange energy density $\varepsilon_{x}[n]$ is plainly sensitive to inaccuracies in the latter. We feel that much work remains to be done on firstprinciples studies of $V_{x}(r)$ via the idempotent Dirac density matrix $\gamma\left(\boldsymbol{r}, \boldsymbol{r}^{\prime}\right)$, which is, of course, the key tool employed in the present study.

\section{ACKNOWLEDGMENTS}

The first author wishes to thank the Department of Theoretical, Atomic and Optical Physics of The University of Val-

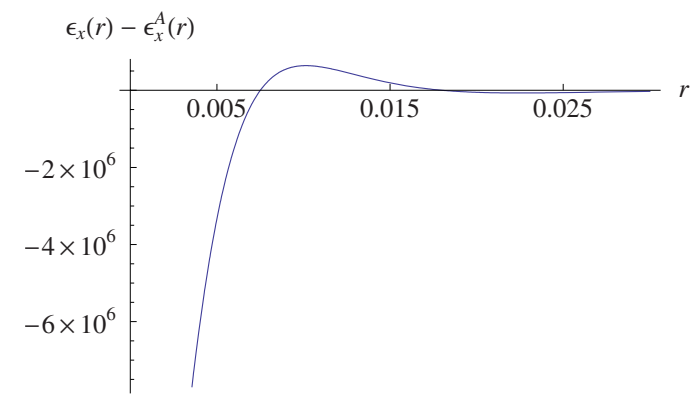

FIG. 13. (Color online) Difference between the exchange energy density $\varepsilon_{x}(r)$ from [8] and alternative form $\varepsilon_{x}^{A}(r)$ in Appendix A for $Z=92$.

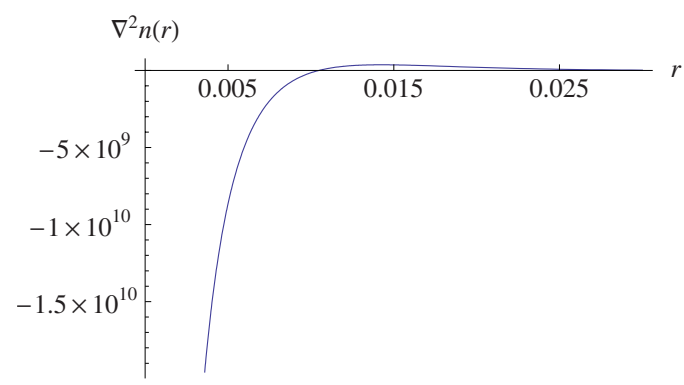

FIG. 14. (Color online) $\nabla^{2} n(r)$ versus $r$ (a.u.) for comparison with the shape of $\varepsilon_{x}(r)-\varepsilon_{x}^{A}(r)$ in Fig. 13 .

ladolid for their hospitality. N.H.M. wishes to acknowledge that his contribution to the present study came to fruition during a visit to the University of Valladolid. Thanks are due to Professor J. A. Alonso and Professor M. Santander for generous hospitality. N.H.M. also had partial financial support from the University of Antwerp through the BOF-NOI. The work of L.M.N. was partially supported by the Spanish Ministerio de Educación y Ciencia (Project No. MTM2009. 10751) and Junta de Castilla y León (Excellence Project No. GR224).

\section{APPENDIX A: ALTERNATIVE EXPRESSION FOR THE EXCHANGE ENERGY DENSITY AT LARGE $Z$}

From Eq. (2.2) of [11], we have, for $r \rightarrow \infty$,

$$
\varepsilon_{x}(r) \rightarrow-\frac{e^{2} \alpha^{3}}{16 \pi r} e^{-\alpha r}(\alpha r)^{2}
$$

But,

$$
n(r) \rightarrow \frac{\alpha^{3}}{4 \pi} e^{-\alpha r} \frac{1}{2}(\alpha r)^{2}
$$

so

$$
\varepsilon_{x}(r) \rightarrow-\frac{e^{2}}{2 r} n(r)
$$

as predicted by March [10].

However, as emphasized above, other definitions are possible for the exchange energy density than the "natural" one

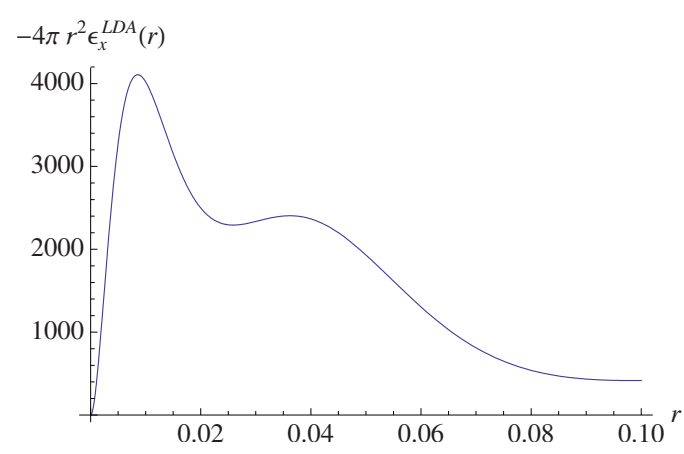

FIG. 15. (Color online) $-4 \pi r^{2} \varepsilon_{x}^{\mathrm{LDA}}$ versus $r$ (a.u.) for the Argonlike ion with $Z=92$. 


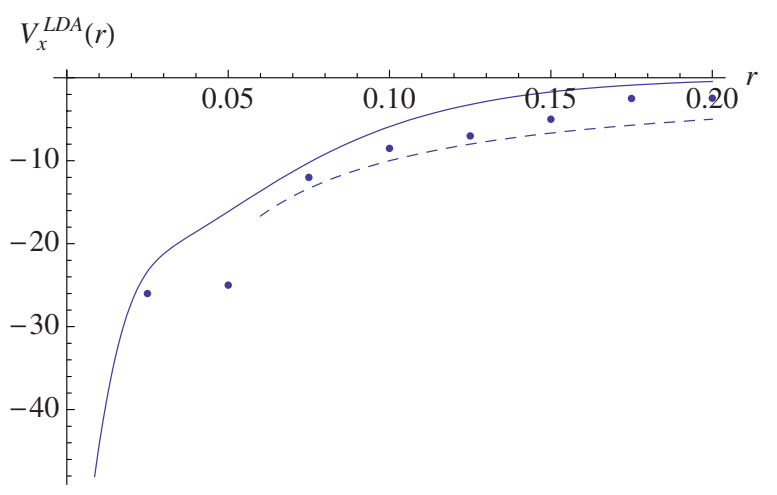

FIG. 16. (Color online) The LDA exchange potential $V_{x}^{\mathrm{LDA}}(r)$ versus $r$ (a.u.) for the Ar-like ion with $Z=96$ (solid curve). The dots are the results from [14] Fig. 4. The dashed curve is the exact asymptotic value for large $r,-e^{2} / r$.

adopted above. Thus we shall write (with A denoting "alternative")

$$
\varepsilon_{x}^{A}(r)=\varepsilon_{x}(r)+\Delta(r)
$$

where $\int \Delta(\boldsymbol{r}) d \boldsymbol{r}=0$ in order to obtain the exact exchange energy [8]

$$
E_{x}=-\frac{489295}{279936}\left(Z e^{2} / a_{0}\right)=-1.74788 Z e^{2} / a_{0} .
$$

In order to adopt a specific choice for $\Delta(\boldsymbol{r})$, we start with Eqs. (3.1) and (3.2) of [8]. Then we can write, almost immediately,

$$
\begin{gathered}
\varepsilon_{x}^{(1) A}(r)=-\frac{5 \pi}{3} e^{2} r^{2} n^{2}(r), \\
\varepsilon_{x}^{(2) A}(r)=-\frac{224 \pi^{2}}{9} r^{4} n(r) F(r),
\end{gathered}
$$

where [12]

$$
F(r)=\frac{n^{\prime \prime}(r)}{24}-\frac{m t(r)}{4 \hbar^{2}}
$$

Here $t(r)$ is the kinetic-energy density which, for the present model, satisfies the Amovilli-March equation [17],

$$
\frac{\partial t(r)}{\partial r}=\frac{\hbar^{2}}{8 m} n^{\prime \prime \prime}(r)-\frac{3 \hbar^{2} n^{\prime}(r)}{4 r^{2} m}-\frac{3 Z e^{2} n(r)}{2 r^{2}} .
$$

It is important to stress that, although the theorems of DFT assure us that

$$
t(r) \equiv t[n(r)],
$$

in fact the simple form of Eq. (A9) is reached by involving the Coulomb force $-\partial V(r) / \partial r$ with $V(r)=-Z e^{2} / r$, where

$$
\frac{\partial V(r)}{\partial r}=\frac{Z e^{2}}{r^{2}} \text {. }
$$

Finally

$$
\varepsilon_{x}^{(3) A}(r)=-8 \pi e^{2} F^{2}(r) r^{6} .
$$

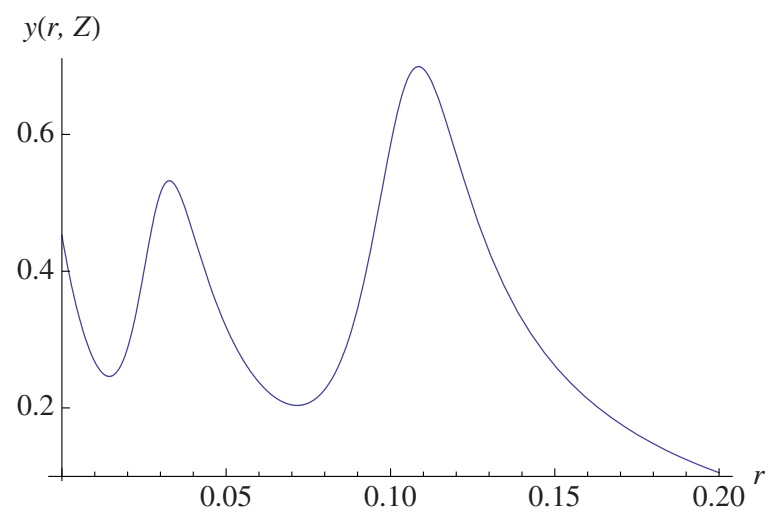

FIG. 17. (Color online) Dimensionless ratio $y(r, Z)$ defined in Eq. (9) for the Ar-like ion with $Z=92$.

Figures 9, 10, and 12 show plots of Eqs. (A6), (A7), and (A12), respectively. Figure 11 shows their sum, $\varepsilon_{x}^{A}(r)$ compared with Eq. (2.2) in [8].

It is readily verified that although $\varepsilon_{x}^{A}(r)$ falls off much faster than $\varepsilon_{x}(r)$ at large $r$, with the precise asymptotic form (A3) given above, the volume integrals agree (to within $1 \%$ due to round-off error). We note that one particular choice of $\Delta(r)$ in Eq. (A4) which has zero volume integral is

$$
\Delta(\boldsymbol{r})=\text { const } \times \nabla^{2} n(\boldsymbol{r}) .
$$

We show below in Figs. 13 and 14 a plot of the difference between $\varepsilon_{x}(r)$ from [8] and $\varepsilon_{x}^{A}(r)$ and a plot indicating the shape of $\nabla^{2} n(r)$. There is some shape similarity and the positions of the nodes may be brought into coincidence by using a scaled normalized density $n_{\lambda}(r)=\lambda^{3} n(\lambda r)$ and forming the Laplacian of $n_{\lambda}(r)$.

\section{APPENDIX B: GROUND-STATE ELECTRON DENSITY FOR ARGONLIKE ATOMIC IONS IN THE LIMIT OF LARGE ATOMIC NUMBER $Z$ AND LDA EXCHANGE POTENTIAL COMPARED WITH OPTIMIZED EXCHANGE RESULT}

This appendix is motivated by the calculation of the OEP for the Argonlike series of atomic ions in the limit of large $Z$ (see [14] Fig. 4). The ground-state density $n_{s}(r)$ is readily obtained from the March-Santamaria [9] density $n_{\mathrm{MS}}(r)$ as

$$
n_{s}(r)=n_{\mathrm{MS}}(r)+2 \psi_{3 s}^{2}(r)+6 R_{31}^{2}(r),
$$

where $\psi_{\mathrm{ns}}(r)$ denotes the normalized $s$-state wave function for the closed shell of principal quantum number $n$ while $R_{31}(r)$ is the radial wave function for the $n=3 p$ state. The $p$-state density, i.e., $n_{2 p}(r)+n_{3 p}(r)$ is given by using the radial wave functions $R_{21}(r)$ and $R_{31}(r)$ [18]. We have

$$
\begin{aligned}
n(r, Z, N=18)= & n_{\mathrm{MS}}(r) \\
& +\frac{Z^{3}}{486 \pi a_{0}^{3}}\left(6-4 Z r / a_{0}+4 Z^{2} r^{2} / 9 a_{0}^{2}\right)^{2} e^{-2 Z r / 3 a_{0}} \\
& +n_{3 p}(r)
\end{aligned}
$$

and $[18]$ 


$$
\begin{aligned}
n_{3 p}(r) & =2\left[\psi_{310}^{2}(\boldsymbol{r})+2\left|\psi_{311}(\boldsymbol{r})\right|^{2}\right] \\
& =6 R_{31}^{2}(r)=\frac{1}{96 \pi}\left(\frac{2 Z}{3 a_{0}}\right)^{2} \rho^{2}(\rho-4)^{2} e^{-\rho}, \quad \rho=\frac{2 Z}{3 a_{0}} r .
\end{aligned}
$$

Figure 15 shows the LDA radial exchange energy density $4 \pi r^{2} \varepsilon_{x}^{\mathrm{LDA}}(r)$ as a function of $r$ for $Z=92$, while Fig. 16 plots the corresponding exchange potential

$$
V_{x}^{\mathrm{LDA}}(r)=-\frac{4}{3} c_{x}[n(r)]^{1 / 3}
$$

for comparison with $V_{x}^{\mathrm{OEP}}(r)$, which is estimated from Fig. 4 of [14]. In this plot we also show the asymptotic value $e^{2} / r$. To complete this appendix, we plot the Becke-type quantity $y(r, 92)=[n(r)]^{4 / 3} /|\nabla n(r)|$ in Fig. 17.
[1] See, for example, N. H. March and C. Van Alsenoy, Phys. Chem. Liq. (to be published).

[2] F. Della Sala and A. Görling, J. Chem. Phys. 115, 5718 (2001).

[3] I. A. Howard and N. H. March, J. Chem. Phys. 119, 5789 (2003).

[4] J. C. Slater, Phys. Rev. 81, 385 (1951).

[5] W. Kohn and L. J. Sham, Phys. Rev. 140, A1133 (1965).

[6] P. A. M. Dirac, Proc. Cambridge Philos. Soc. 26, 376 (1930).

[7] L. J. Kleinman, Phys. Rev. B 49, 14197 (1994).

[8] I. A. Howard, N. H. March, P. Senet, and V. E. Van Doren, Phys. Rev. A 62, 062512 (2000).

[9] N. H. March and R. Santamaria, Phys. Rev. A 38, 5002 (1988).
[10] N. H. March, Phys. Rev. A 36, 5077 (1987).

[11] F. Herman, J. P. Van Dyke, and I. B. Ortenburger, Phys. Rev. Lett. 22, 807 (1969).

[12] J. C. Stoddart, P. Stoney, N. H. March, and I. B. Ortenburger, Nuovo Cimento 23B, 15 (1974).

[13] A. D. Becke, Phys. Rev. A 38, 3098 (1988).

[14] I. A. Howard, N. H. March, and J. D. Talman, Phys. Rev. A 68, 044502 (2003).

[15] M. Levy and J. P. Perdew, Phys. Rev. A 32, 2010 (1985).

[16] A. Nagy and N. H. March, Phys. Chem. Liq. 37, 671 (1999).

[17] C. Amovilli and N. H. March, Phys. Chem. Liq. 38, 505 (2000).

[18] L. Pauling and E. B. Wilson, Jr., Introduction to Quantm Mechanics (McGraw-Hill, New York, 1935). 